\title{
Horizontale und vertikale Achsen in der vorchristlichen skandinavischen Kosmologie
}

\author{
Von Jens Peter SchJødt
}

Das Ziel der folgenden Untersuchung ist es, einige Faktoren zu erhellen, die für unser Verständnis des Weltbildes von Bedeutung sind, so wie es vermutlich in der Wikingerzeit vor der Einführung des Christentums ausgesehen hat. Einige der Konsequenzen, die die Auffassung von diesem Weltbild für unser Verständnis der nordischen Religion und Mythologie generell mit sich bringt, sollen ebenfalls untersucht werden. Die meisten Forscher haben der Tatsache Aufmerksamkeit geschenkt, dass unsere Quellen in diesem Punkt offenbar eine Reihe widersprüchlicher Informationen liefern, während die Erklärungen zur Erhellung dieser Widersprüche ein ausgesprochen weites Spektrum aufweisen.

In dieser Untersuchung soll vor allem das Verhältnis zwischen den sogenannten vertikalen und horizontalen Subsystemen in dem räumlichen Modell der Skandinavier diskutiert werden, um die Terminologie zu benutzen, die E. M. Meletinskij in seinem berühmten Artikel von 1973 "Scandinavian Mythology as a System" I-II (Meletinskij 1973a, $46 \mathrm{ff}$.$) angewandt hat. Man hat in der Kosmologie mit zwei Achsen$ gearbeitet, einer vertikalen, mit den Polen Himmel-Unterwelt und einer horizontalen, mit den Polen Miðgarðr (oder Ásgarðr)-Útgarðr. Das Widersprüchliche liegt hauptsächlich in dem Standort, der den Göttern in den beiden Modellen zugeordnet wird: in dem vertikalen befindet sich ihr Platz im Himmel, während sie in dem horizontalen ihren Platz in Ásgarðr haben, im Zentrum der Welt am Fuss der Weltesche Yggdrasill.

Die Quellen, die primär benutzt wurden, um die heidnische Kosmologie zu rekonstruieren, sind Die ältere und Die jüngere Edda, aber es sind natürlich auch andere hinzugezogen worden. Gylfaginning und Grimnismál sind besonders explizit in der Darstellung des Weltbildes. Es steht fest, dass Gylfaginning um 1220 verfasst wurde, während die Datierung von Grimnismál beträchtlich variiert. Die meisten jedoch datieren es zurück in die heidnische Zeit, was ganz angemessen zu sein scheint (z. B. Vries 1964-67, 1, 45; Stanley Martin 1972, 20 f.). 
Snorri bietet uns einen gewaltigen Wirrwarr an Informationen über die Einrichtung des Kosmos. In Kap. 6 wird erzählt, dass die Götter Miðgarðr aus Ymirs Wimpern gebaut haben, was zuerst eine Burg genannt wird, während kurz danach gesagt wird, dass die Götter Ásgarðr in der Mitte der Welt gebaut haben, worin sie und ihre Familien dann gewohnt haben. Kurz davor wurde uns in demselben Kapitel etwas über die Form der Welt erzählt: sie ist rund und von einem tiefen Meer umgeben. An den Küsten dieses Meeres wohnen die Riesen, während die Menschen weiter im Inneren wohnen. Es ist bemerkenswert, dass aus drei Handschriften (RWT) hervorgeht, dass Ásgarðr von den Menschen Troja gen annt und somit als irdischer Ort aufgefasst wird - ein Zug, der ein Ausdruck von Snorris Euhemerismus sein kann, hinter dem aber auch mehr liegen könnte. Wir befinden uns ebenfalls auf der horizontalen Achse, wenn davon berichtet wird, dass es östlich von Miðgarðr einen Wald gibt, der Iárnviðr heisst, wo eine Riesin ihre Brut zur Welt bringt. Diese Zuordnung der Riesen im Osten findet man bei Snorri und in anderen Quellen häufig. Am Schluss des Kapitels wird von Bifrosst erzählt, die laut Snorri ein Regenbogen, aber gleichzeitig auch eine Brücke ist, die Himmel und Erde miteinander verbindet, was hier ein wenig verwunderlich ist, denn nach dem, was wir bisher gehört haben, sind die Götter eindeutig in der Weltmitte auf der horizontalen Achse angesiedelt. In Kap. 7 befinden wir uns bei der Beschreibung der Heiligtümer der Götter wieder auf dieser Achse, wo Glaðsheimr als das beste und grösste Haus der Welt bezeichnet wird. Dagegen ist die im gleichen Kapitel beschriebene Schöpfung der Zwerge und deren in Erde und Steinen gelegene Wohnstätte vertikal orientiert.

In Kap. 8 berichtet Snorri über Yggdrasill. Dort ist der Hauptort oder auch die heilige Stätte der Götter, und hier halten sie jeden Tag Gericht. Er ist der grösste aller Bäume und ragt hoch in den Himmel hinein, während seine drei Wurzeln nach drei Seiten gehen - zu den Asen, den Reifriesen und zu Hel. Die Wurzel, die zu den Asen führt, befindet sich im Himmel, was ziemlich ungewöhnlich erscheint. Aber jetzt bekommt Bifrosst seine natürliche Erklärung, denn die Asen reiten jeden Tag darüber, um zu ihrem Gerichtshof zu kommen. Nach noch einer eingehenden Beschreibung von Yggdrasill erfährt man, dass man unter der Wurzel der Esche bei den Göttern Urds Brunnen (Urðar brunnr) finden kann. In Kap. 9 fragt Gangleri in folgender Weise: "Grosse Nachricliten kannst du über den Himmel kundtun. Welche anderen Hauptorte mag es wohl ausser Urds Brunnen dort geben?" Urds Brunnen befindet sich also im Himmel, genauso wie die folgenden Orte, die die Antwort auf die gestellte Frage darstellen: Alfheimr, 
Breiðablik und viele andere Wohnstätten der Götter. Am südlichen Ende des Himmels befindet sich z. B. Gimlé, aber etwas später wird gesagt, dass sich dieser Ort in dem dritten Himmel befindet - der also nicht identisch ist mit dem Himmel, den wir sehen können. Auch die Entstehung des Windes wird mit Hinweis auf eine Himmelsrichtung, nämlich Norden, erklärt: dort sitzt Hræsvelgr und schlägt so mit den Flügeln, das Wind entsteht. Snorri belegt seine Information mit Vm 37, wo jedoch nichts vom nördlichen Ende des Himmels gesagt wird. In Kap. 11 wird davon erzählt, dass Baldr und Njgrðr im Himmel wohnen, genauso wie wir es später über verschiedene andere Götter erfahren. In Kap. 19 und 20 erfahren wir in Verbindung mit den Kindern von Loki, dass die Welt von der Midgardschlange umgeben ist, während Hel in den Niflheimr (im übrigen ein Name, der in den älteren mythologischen Liedern nicht vorkommt) geworfen wird. Die Zuordnung der Riesen nach Norden im horizontalen Modell wird von folgendem Bild bestärkt: von Hliðskiálf aus sieht Freyr im Norden die Riesin Gerðr. Zum Schluss soll noch kurz eine von Snorris Aussagen über die mythologische Geografie erwähnt werden; in Kap. 34 beschreibt er, dass der Weg nach Hel nach Norden und dann nach unten führt, wodurch Hel dann beiden Ausrichtungen zugeordnet wird der horizontalen und der vertikalen. Man könnte in Gylfaginning noch zahlreiche andere Beispiele anführen, die Aussagen über die Einrichtung des Kosmos machen, aber die erwähnten Beispiele dürften genug sein, um sich einen Eindruck davon zu verschaffen, wie chaotisch diese Informationen im Verhältnis zu einem ein deutigen Kosmosmodell sind.

Bevor ich noch auf andere Quellen eingehe, scheint es mir angemessen, einen Blick darauf zu werfen, was andere Forscher mit diesem Weltbild anfangen können, oder besser gesagt mit diesen Steinchen eines Mosaiks, die ein Weltbild ausmachen. Wie gesagt, haben die meisten der Tatsache Aufmerksamkeit geschenkt, dass mit verschiedenen Kosmosbildern gearbeitet wird, denn die Inkonsequenzen bei Snorri sind nicht gerade schwer zu entdecken (vgl. z. B. Gurevich 1969, 46). Es ist also notwendig, für diesen Mangel an Eindeutigkeit eine Erklärung zu finden, und die verschieden Erklärungsversuche weisen eine ausgesprochen grosse Variationsbreite auf. Dennoch wollen wir es hier wagen, diese Versuche in zwei Kategorien einzuteilen, deren Zugang zur Problematik grundsätzlich verschieden ist - nämlich eine historisch orientierte und eine strukturalistisch orientierte. Einige wenige Beispiele dieser verschiedenen Zugangsmöglichkeiten dürften zur Erläuterung ausreichen. Jan de Vries beschreibt z. B. die zwei Weltbilder - das vertikale und das horizontale - auf etlichen Seiten, jedoch 
ohne diese Terme explizit zu gebrauchen und fast ohne sie aufeinander zu beziehen (Vries 1956-57, 2, 372 ff.). Er schreibt: "Der Himmel ist der Wohnort der Götter, jedenfalls nach der späteren Vorstellung. Die Götterwohnungen, die Ásgarðr bilden, werden schliesslich nicht mehr auf Erden, in der Nähe der Menschenwelt, gedacht, sondern in den Himmel versetzt." Auch Neckel hatte bereits im Jahre 1913 eine ähnliche Auffassung, nämlich dass Walhall zu einem späten Zeitpunkt in heidnischer Zeit in den Himmel gerückt wird (z. B. Neckel 1913, 56 ff.), wohingegen Odins Platz im Himmel als uralt dargestellt und auf indoeuropäische Zeit zurückdatiert wird (Neckel 1913, 59 ff.). Neckels Darstellung ist lang, kompliziert und wenig wahrscheinlich, aber charakteristischerweise nimmt er ganz selbstverständlich an, dass der Platz der Götter in spätheidnischer Zeit im Himmel war.

Neckel und de Vries und vielen anderen ist es gemeinsam, dass die Widersprüche in den Quellen historistisch erklärt werden. Demgegenüber ist es interessant, die Versuche einer Reihe von Forschern zu beobachten, die eine ganz andere Methode zur Erklärung dieser Dinge heranziehen. Es scheint in den neueren Versuchen, die nordische Mythologie zu beschreiben, geradezu eine Tendenz dazu vorhanden zu sein, in wesentlich höherem Masse gerade die Kosmosvorstellungen mit einzubeziehen, was als solches schon als positiv betrachtet werden muss. Diese neueren Forscher haben natürlich auch ihre Aufmerksamkeit auf die verschiedenen offenbar widersprüchlichen Informationen gerichtet, die unsere Quellen in Verbindung mit dem Weltbild bieten, aber im Gegensatz zu den älteren, historistisch orientierten Untersuchungen spielen hier verschiedene Aspekte der strukturalistischen Analysestrategie eine grosse Rolle. Anstatt die verschiedenen Ideen altersmässig einzuordnen, worin z. B. Neckel und seine Generation schwelgten, strebt man danach, die Logik der Vorstellungswelt zu verstehen, die uns unsere Quellen vermitteln. Es soll hier gleich gesagt werden, dass dieses "research-paradigm" meine volle Sympathie hat und dass es die Frage nach Sinn und Bedeutung in wesentlich höherem Masse als das historistische Projekt scheint beantworten zu können. Andererseits besteht darin auch eine Gefahr, nicht zuletzt auf einem Gebiet wie dem nordischen, wo der Hauptanteil unserep Quellen nach einem Religionswechsel verfasst wurde, der jedenfalls in einiger Hinsicht einige ausgesprochen bedeutungsvolle Veränderungen für Individuum und Gesellschaft mit sich gebracht hat. Wie dem auch sei, es gibt besonders drei Forscher, deren Untersuchungen sich in starkem Masse um diese horizontal/vertikale Problematik zentrieren, weshalb deren Ansichten in diesem Zusammenhang näher diskutiert werden 
sollen. Es handelt sich um E. Meletinskij: "Scandinavian Mythology as a System" von 1973, Kirsten Hastrup: "Cosmology and Society in Medieval Iceland" von 1981 und Henk Molenaar: OJinns Gift von 1985. Diese Analysen umfassen - nicht zuletzt in Verbindung mit den Kosmosvorstellungen - jede für sich ausgesprochen spannende Resultate, die trotz der Kritik, die an ihnen hier geübt werden soll, und die sich ganz allein auf die Bedeutung bezieht, die sie Snorris Informationen über die Götterwelt im Himmel zulegen, wertvoll sind.

Meletinskij, auf den ich später näher eingehen werde, ist ganz explizit bei der Unterscheidung zwischen den beiden Kosmossystemen, die er als zwei räumliche Subsysteme beschreibt, deren Inhalt von dem einen "Kode" zum anderen "konvertiert" werden kann (Meletinskij 1973a, 46). Über Yggdrasill wird behauptet, dass dieser die Oppositionen zwischen den Göttern und den chthonischen Mächten auf der vertikalen Achse kennzeichnet. Er schreibt: "The meeting of the gods take place at the top of the tree, their dwellings (Asgard) are also found there..." (Meletinskij 1973a, 49). ${ }^{1}$ Danach analysiert Meletinskij eine Reihe von Elementen auf den beiden Achsen und versucht sie u.a. in Relation zueinander zu setzen. Die Hervorhebung der beiden Subsysteme ist wesentlich für Meletinskijs Projekt als solches, weil ihnen zwei Zeitauffassungen entsprechen: eine reversible und eine irreversible - eine Behauptung, die weiter unten ausfürlich diskutiert werden soll. In diesem Zusammenhang ist es jedoch entscheidend, dass die himmlische Zuordnung der Götter im heidnischen Selbstverständnis nicht in Frage gestellt wird.

Die dänische Anthropologin Kirsten Hastrup ist von Meletinskijs Ansichten stark beeinflusst, baut sie aber auf höchst interessante Weise aus: u.a. indem sie in Verbindung mit dem horizontalen Modell einen anderen sowjetischen Forscher, A. Ya. Gurevich, und dessen Parallelisierung der makro- und mikrokosmischen Vorstellungen mit einbezieht (Hastrup 1981, 64 f.; Gurevich 1969, 42 ff.). Für uns ist jedoch in diesem Zusammenhang Hastrups Übernahme und Ausbau von Meletinskijs Theorien des vertikalen Modells von Bedeutung. Sie schreibt: "On top of the tree was the abode of the Gods, Ásgaror, and Valhalla, the upper kingdom of the dead ruled over by Orinn. [...] The middle of the world-ash was the place of the humans, while at its roots we find the lower kingdom of the dead, ruled over by the goddess

\footnotetext{
1 An dieser Stelle ist es wichtig, darauf aufinerksam zu machen, dass Meletinskij in seinem Artikel nicht Snorris Weltbild analysiert, wie es in seiner Edda zum Ausdruck kommt, sondern generell über "eddic" Mythologie als ein System redet und darüber, wie es in heidnischer Zeit konzipiert war. (Meletinskij 1973a, 43).
} 
Hel" (Hastrup 1981, 66). Hastrup benutzt diese vertikal orientierte Kosmologie, um eine Reihe von Oppositionspaaren aufzustellen, die sich alle analog zu der hoch-niedrig Opposition verhalten. Bei Hastrup finden wir ausserdem eine Vertiefung von Meletinskijs etwas wager Aussage über das Verhältnis zwischen Reversibilität und Irreversibilität einerseits und den zwei räumlichen Modellen anderseits. Wir sehen also, dass die Feststellung der beiden Modelle bei Hastrup die gleiche Bedeutung für die Logik der heidnischen Ideologie hat wie bei Meletinskij, aber die Einordnung der Götter in den Himmel hat hier noch grössere Bedeutung bei der Auslegung der vertikalen Trichotomie und ihrer entsprechenden horizontalen Parallele (Hastrup 1981, 67).

Zum Schluss soll die Dissertation des Holländers Henk Molenaar von 1985 erwähnt werden, die in vieler Hinsicht einen erfrischenden neuen Denkansatz für die gesamte nordische Mythologie bedeutet, die jedoch grosse Mängel aufweist, u.a. was die grundlegenden Kenntnisse der mehr philologisch orientierten Forschung betrifft. Molenaar geht, wie die anderen, die oben erwähnt wurden, wie selbstverständlich davon aus, dass Walhall im Himmel liegt (Molenaar 1985, 71), und auch er versucht, die Oppositionspaare von der einen Achse auf die andere zu "überführen", genauso wie er dem Verhältnis zwischen den beiden Zeitmodellen grosse Bedeutung beimisst (Molenaar 1985, 92).

Die drei Forscher haben also gemeinsam, dass sie es nicht nur als selbstverständlich betrachten, dass Ásgarðr und Walhall nach heidnischer Auffassung im Himmel lagen - auch spielt diese "Feststellung" in ihren weiteren Analysen der gesamten heidnischen Vorstellungswelt eine grosse Rolle.

Wie bereits erwähnt haben alle drei Forscher eine Reihe interessanter Ideen, aber dennoch scheint es angemessen zu untersuchen, ob diese grundlegende Annahme einer näheren Analyse standhalten kann, da alle drei Forscher sich anscheinend alleine auf Snorris Aussagen stützen.

In Grm sowie in der übrigen Götterdichtung der älteren Edda und was das betrifft auch in der Skaldendichtung bis hin zum Jahr 1000 sind die Informationen eher fragmentarisch, aber abgesehen von einigen wenigen Strophen, die aller Wahrscheinlichkeit nach christlich beeinflusst sind, ist es charakteristisch, dass Snorris gesamte himmlische Konstruktion gänzlich unbekannt ist. ${ }^{2}$ Wenn die Wohnstätten

2 Man könnte Vsp 41 als Beweis dafür anführen, dass "ragna sjgt" eine Kenning für den Himmel ist, was der Meinung von fast allen Interpreten entspricht. Mir scheint es jedoch kaum überzeugend zu sein. Es ist zwar (vermutlich) die Rede 
der Götter im Himmel wirklich ein so fester Bestandteil im heidnischen Weltbild gewesen wären, wie Snorris Bericht es uns vermuten lässt, dann ist es gelinde gesagt auffallend, dass keines der vermutlich älteren Gedichte überhaupt darauf eingeht. Dies gilt besonders für $V s p, V m$ und $G r m$, die sich alle recht ausführlich den verschiedenen Phänomenen bei der Einrichtung des Kosmos widmen. In diesem Zusammenhang soll kurz erwähnt werden, dass Strophe 65 in Vsp, die nur im Hauksbók vorkommt, und vermutlich auch Strophe 64 eindeutig christlich inspiriert sind, seien sie nun spätere Interpolationen oder mit dem Gedicht um die Jahrtausendwende entstanden. In allen diesen Gedichten befinden wir uns ganz klar in dem horizontalen Modell. Gute Beispiele bieten $V m$ 16, wo wir erfahren, dass der Fluss Ífing die Gebiete der Riesen und der Götter voneinander trennt, und Grm 29, worin pórr nachgesagt wird, dass er die verschiedenen Flüsse durchwatet, um zu Yggdrasill zu gelangen, weil die Asenbrücke (die mit Bilrost identisch sein muss) in Flammen aufgeht. Viele andere Beispiele könnten noch genannt werden, die mehr oder weniger stark indizieren, dass wir es eindeutig mit einer horizontal orientierten Kosmosauffassung zu tun haben. Selbst in den Beschreibungen von Yggdrasill in Vsp und Grm gibt es nicht die geringste Andeutung einer

von dem Wolf, der die Sonne verschlingt, aber hier in der ersten halben Strophe ist er eindeutig als ein Leichenwolf beschrieben, der das Blut der Gefallenen aufsaugt, und in Verbindung mit der herannahenden Ragnarok-Schlaclit, wo das Schlachtfeld nahe den Wohnstätten der Götter liegt, ist es ganz natürlich, dass sie von diesem Blut rot gefärbt werden. Dass die Sonme in der zweiten halben Strophe schwarz wird, ist - trotz Nordals poetischem Erklärungsversuch (Nordal 1927, 81) eher darauf zurückzuführen, dass die Sonne geraubt wurde (vgl. Strophe 40, wo der Wolf als "tungls tjúgari" beschrieben wird, also als jemand, der etwas entfernt). Was $\mathrm{HH}_{2} 49$ betrifft, wo ein "flugsstigr" (ganz gleich welche Übersetzung man vorzieht, vgl. Sijmons \& Gering 1906-31, 3, 2, 132, so ist es mit einem Himmelsweg verbunden worden) und einige Himmelsbrücken - "Vindhjalms brúar" erwähnt werden, so können diese keineswegs als Beweis für eine limmlische Wohnstatt genommen werden. Hingegen können sie, worauf unten noch näher eingegangen wird, als Beispiele dafür gelten, dass die Luft oder der Himmel als Transportweg fungierten.

Schiesslich soll Hliðskjálf erwähnt werden, der oft als Hochsitz in einem himmlischen Walhall betrachtet wird (z. B. in der einleitenden Prosa zu Grm und Skm), von wo aus man in alle Welt schauen kann. Hierzu kann man bloss sagen, dass das "himmlische" an Hlidskjalf nur in Erscheinung tritt, wenn man Snorris Weltbild mit in Betracht zieht. Erstens kann man sich vorstellen, dass dieser Sitz magische Kräfte besitzt (Kiil 1960), die einem ermöglichten, in die ganze Welt zu schauen, und zweitens muss man sich Walhall a priori hochangebracht in der Mitte der Welt vorstellen, was eine gewisse Aussicht erinöglicht. 
himmlischen Wohnstatt für die Asen.

Nun kann man behaupten, dass ein grosser Teil der neueren Forschung, wobei hier besonders Dumézil und seine Schüler genannt werden sollen, gezeigt hat, dass es eine Menge mythischen Stoff gibt, den man nur von Snorri kennt, der aber dennoch uralt sein muss. Dies ist zweifellos richtig, aber es muss doch festgehalten werden, dass es zwischen einzelnen Mythen oder mythischen Details und einer so grundlegenden Sache wie dem Standort der Götter einen Unterschied gibt. In Relation gesehen zu dem vielen Platz, den der Himmel in Gylfaginning einnimmt, wirkt das Schweigen der anderen Quellen fast lärmend. Es scheint daher ganz unwahrscheinlich zu sein, dass ein Gedicht wie Grm, das sich im grossen und ganzen mit nichts anderem als mythischen Örtlichkeiten beschäftigt, einen solchen himmlischen Standort nicht weitergegeben oder wenigstens angedeutet hätte, wenn es dem Dichter bekannt gewesen wäre. Das gleiche gilt für die Kenningkunst, wo nichts uns vermuten lässt, dass die Götter "himmlisch" gewesen sind, in dem Sinne dass sie auch im Himmel gelebt haben.

Es ist hier keineswegs die Absicht, Snorris Angaben, so wie er sie in Gylfaginning darstellt, hinsichtlich einer Unterscheidung zwischen heidnisch und christlich zu sezieren. Dies ist bereits oft unternommen worden, und hatte in vielen Fällen ein unangemessenes Misstrauen Snorri gegenüber zur Folge. Dennoch scheint es mir an dieser Stelle angemessen, auf Anne Holtsmarks in vieler Hinsicht überzeugendes Werk Studier i Snorres mytologi hinzuweisen, das besonders bezüglich der Kosmosvorstellungen gezeigt hat, wie sehr Snorri von seinem christlichen Kinderglauben beeinflusst war (Holtsmark 1964, 27 ff., 55 ff.), den er natürlich mit seiner Kenntnis der heidnischen Ideologie kombiniert hat.

Auch Hastrup führt übrigens den Gedanken aus, dass es beträchtliche Åhnlichkeiten zwischen dem heidnischen "vertikalen" Modell und der mittelalterlichen christlichen Kosmosauffassung gibt, kommt aber nicht auf die Idee zu fragen, ob es überhaupt so etwas wie ein vorchristliches räumliches Modell gegeben hat, wo die Götter "himmlisch" waren. Zieht man das Quellenmaterial in Betracht, so ist das wie gesagt nicht besonders wahrscheinlich, und die Ähnlichkeiten zwischen den beiden vertikalen Modellen, dem christlichen und dem "vorchristlichen" lassen sich am besten dadurch erklären, dass die himmlischen Wohnstätten der Götter ganz einfach ein postheidnisches Phänomen sind. Daher kann ich voll und ganz Gurevichs Ansicht unterstützen, der in dem oben bereits genannten Artikel sagt: "... there is no reason to suppose that the Scandinavians imagined their gods to be inhabi- 
tants of some heavenly spheres" (Gurevich 1969, 46). Es erscheint recht unverständlich, dass weder Hastrup noch Molenaar diese Aussage nicht im geringsten reflektiert haben, obwohl sie beide den Artikel mit einbezogen haben; dies ist besonders zu beklagen, da er wie gesagt mit dem entsprechenden Quellenmaterial in völliger Übereinstimmung steht.

Warum so viele Forscher, ohne auch nur einen Ansatz von quellenkritischem Lesen zu zeigen, Snorris himmlisches Götterheim akzeptiert haben, darüber können natürlich nur Vermutungen angestellt werden; aber man kann sich nicht von dem Verdacht lösen, dass sich die betreffenden Forscher, genau wie Snorri, nur schwer von ihrem Kinderglauben lösen konnten, dass "richtige" Götter in den Himmel gehören. ${ }^{3}$

Dass es dennoch in gewisser Weise angemessen ist, in der vorchristlichen nordischen Religion mit einer vertikalen Achse zu arbeiten, jedoch ohne im Himmel wohnende Götter, darauf werde ich später noch zurückkommen. Aber vorher wollen wir noch auf einige weitere, eher positive Argumente eingehen, um der Frage nachzugehen, warum die Skandinavier schwerlich diese Vorstellung gehabt haben können.

Gurevich (Gurevich 1969, 42 ff.) und Hastrup (Hastrup 1981, 65, $69 \mathrm{ff}$.$) zeigen, von einer überwiegend soziologischen Argumentation$ ausgehend, dass die "world-view", die dem horizontalen räumlichen Modell zu eigen ist, in verblüffender Weise auch den sozialen Raum mit Zentrum und Periferie geprägt hat. Ihre Argumentation, auf die hier nicht näher eingegangen werden soll, scheint überzeugend und entspricht übrigens ausgezeichnet den Vorstellungen der Religionsphänomenologie über Mikrokosmos und Makrokosmos. Aber auch was die Auffassung der Beziehungen zwischen Göttern und Menschen angeht, die in unseren Quellen zum Ausdruck kommt, scheint es wesentlich natürlicher, Götter und Menschen auf derselben horizontalen Achse anzusiedeln. Das, was zuerst ins Auge fällt, sind eine Reihe von Grundbedingungen, die für Götter und Menschen gleich sind; hierzu

3 Die Vorstellung, dass die Götter im Himmel wohnen, ist natürlich keine spezifisch christliche, sondern ist im Gegenteil auf der ganzen Welt verbreitet. Aber dennoch haben die meisten Religionen auch Götter, die man nicht dort findet, sondern in der Unterwelt, in den Bergen, in den Wäldern etc. Das klassische Beispiel dafür ist natürlich der Olymp, also ein Ort auf der Erde, den man sich als Aufenthaltsort der griechischen Götter vorgestellt hat (auch selbst wenn Zeus, schon allein wegen der Etymologie des Namens, Relationen zum Himmel gehabt haben muss. Wie man dies historisch erklären will, ist diskutabel, aber in der klassischen und archaischen Periode ist die "irdische" Position indiskutabel). 
gehört vor allem die Schicksalsgemeinschaft: Ragnarok würde eine, wenngleich nicht totale, so doch fast gänzliche Destruktion beider "Geschlechter" bedeuten. Daher ist ihr Verhältnis zu den Chaosmächten gleich; beide müssen sich gegen sie schützen - und dies keineswegs in irgendeinem ethischen Sinne, wo für die eine oder andere Seite Partei ergriffen wird, sondern im ganz konkreten Sinne durch Kampf. Der Unterschied z wischen Göttern und Menschen ist eher quantitativer als qualitativer Art.

Man könnte eine grosse Anzahl Mythen aufzählen, die den "menschlichen" Charakter der Götter deutlich werden lassen, aber hier soll es genug sein, an die Prosaeinleitung zu Reginsmál oder an Óðinn's Erlebnisse mit Billings mær in Hávamál zu erinnern. Denn auch wenn bórr eindeutig stärker ist als die meisten und auch wenn Óðinn's magische Kräfte die der Menschen ïbertreffen usw., so kann hier doch nur die Rede von feinen Gradunterschieden sein, die keinesfalls dazu berechtigen, die Götter auf ein ganz anderes Niveau als die Menschen zu plazieren. Es ist also in der skandinavischen Gott-MenschenRelation keineswegs von der Opposition vollkommen vs unvollkommen die Rede. Das Verhältnis impliziert ebenfalls eine gewisse Interaktion, die jedoch nie auf der vertikalen Achse nach oben hin stattfindet. Pórr macht z. B. in Miðgardr in Hym halt und bekommt hier pjalfi und Roskva übereignet. Diese können andererseits auch ohne weiteres in Ásgarðr wohnen. In der Heldendichtung hält sich Óðinn oft bei den Menschen auf und steht ihnen mit gutem Rat zur Seite; dies findet man z. B. ebenfalls in Saxo und den Fornaldarsagen. ${ }^{4}$

Ausser der "Gemeinsamkeit des Schicksals", die bei Ragnarok am stärksten impliziert ist, kann ebenfalls die Machtunvollkommenheit genannt werden, denen auch die Götter unterliegen. Óðinn hat z. B. keine Kontrolle über seine Walküre, als sie einen anderen als den von ihm Erwählten gewinnen lässt (Sigrdrífumál), aber er kann sie mit Hilfe von magischen Kräften strafen, was allerdings irdische Zauberer oder Magier ebenfalls vermögen.

All dies kann natürlich nicht "beweisen", dass die Götter ihren Platz nicht im Himmel hatten, aber zieht man gleichzeitig in Betraclit, dass die älteren poetischen Quellen diesen Umstand nie erwähnen, so schei-

4 Bifrost kann nicht als Argument für das Gegenteil gebraucht werden, denn nur bei Snorri kommt es in Form einer Brücke vor, die vom Himmel zur Erde reicht. In Grm 29 wird darüber keine Andeutung gemacht, dass es diese beiden Orte sind, die die Brücke verbindet. Ausserdem kann die Vorstellung von einer Brücke eine spätere Vorstellung sein, die eine ältere Vorstellung von einem eigentlichen "Weg" abgelöst hat, wie einige Forscher meinen (vgl. z. B. Ström, $\AA$. V. 1975, 191). 
nen doch recht starke Indizien darauf hinzuweisen.

Oben ist einzig und allein dafür argumentiert worden, dass die heidnischen Skandinavier höchstwahrscheinlich nie ein Weltbild gehabt haben, wo die Götter ihre Wohnstatt im Himmel hatten. Die Tatsache, dass wie gesagt trotzdem eine vertikale Achse existiert hat, kann auf zweierlei Weise belegt werden. Erstens hat der Himmel als vorübergehender Aufenhaltsort für verschiedene Wesen fungiert, wozu nicht zuletzt Pórr gehört, der hier mit seinem von Böcken gezogenen Wagen entlang fährt. Und zweitens gibt es eine Unmenge von Beispielen für die Existenz einer Achse, die die Oberwelt (Miðgarðr und Ásgarðr) mit der Unterwelt (Hel) verbindet. Die räumliche Vorstellung von einer vertikalen Achse nach unten hin hat zweifellos in dem ideologischen und semantischen Universum der Skandinavier eine grosse Rolle gespielt (vgl. z. B. Schjødt 1983; Motz 1975; Motz 1980), wovon die Edda- und Skaldendichtung ein so reiches Zeugnis ablegt. Es soll hier also keineswegs gegen die Vertikalität als Orientierungsform argumentiert werden, dagegen aber gegen die Ansicht, dass die heidnischen Vorstellungen über die Einrichtung des Kosmos mehr oder weniger chaotisch, und auf jeden Fall widersprüchlich gewesen sind. (Meletinskij 1973a, 46). Genau das habe ich in der vorgehenden Untersuchung versucht zurückzuweisen, und das Kosmosbild, das zum Vorschein kommt, scheint daher eine absolut zusammenhängende Konzeption aufzuweisen: die Götter haben ihren Platz im Zentrum; aussen herum sind dann die Menschen, die Riesen und das Weltenmeer in selbiger Reihenfolge ${ }^{5}$ in "Domänen" plaziert, die als konzentrische Zirkel aufgefasst wurden. In Richtung nach "oben" ist der Himmel, Ymirs Schädel, auf dem die Sterne und andere Himmelskörper sitzen und durch den die Götter durchreisen konnten - besonders pórr, wenn er über Miðgárðr nach Útgarðr und wieder zurück flog. In diesem Sinne sind die Götter natürlich "himmlisch", aber es ist charakteristisch, dass der Himmel in diesem Zusammenhang "benutzt" wird und daher in Relation zu dem horizontalen Modell eine Funktion bekommt. Aber die Fähigkeit, sich durch den Himmel zu bewegen, ist keinesfalls nur den Göttern vorbehalten, denn z. B. Níðhgggr (Vsp 66), der Riese piazzi (Haustlgng) und andere Wesen wie Magier (Vatnsdalasaga Kap. 212)

5 Und nicht, wie Hastrup behauptet, eine Reihenfolge wie folgt: Götter, Menschen, Meer und dann Riesen. In diesem Punkt ist Snorri ganz deutlich (Kap. 6), und es ist auch nicht die Rede davon, dass ein Meer durchfahren werden muss, um zum Land der Riesen zu gelangen. Dass die Riesen (jedenfalls einige von ihren) laut $V s p$ zum Ragnarokkampf segeln, braucht keinensfalls so aufgefasst werden, als sei es Uthaf, das überquert wird. 
bedienen sich des Himmels als Transportweg, wenn sie es eilig haben, und so gesehen sind Riesen und Menschen genauso "himmlisch" wie die Götter. Der Himmel ist sozusagen "leer" gewesen, und diejenigen, die sich darauf verstanden, konnten dort verkehren. Ausserdem soll hier noch festgehalten werden, dass die Richtungsangabe "nach oben" auch die Wohnstätte der Götter gegenüber der Menschenwelt bezeichnet haben kann (z. B. Sonatorrek $21^{b}$ ), ohne dass gleich vom Himmel die Rede sein muss: eine solche Richtungsangabe kann ebenso auf das Zentrum der Welt gerichtet sein, das im Verhältnis zum Meer oben gelegen haben muss. Die vertikale Achse war dagegen in Richtung nach unten dicht mit Wesen bevölkert, die auf irgendeine Art und Weise mit der Unterwelt verbunden waren. Dies gilt für Gruppen wie Zwerge, Elfen und vermutlich auch die Wanen, betrachtet als Gesamtgruppe (Schjødt 1984), aber hauptsächlich wohl für Hel und die Toten, die in ihrem Reich verkehren. Ganz abgesehen davon wie interessant diese Unterwelt ist, so soll es in diesem Zusammenhang ausreichen, auf ihre Existenz hinzuweisen, wodurch die vertikale Achse dann etabliert ist. Aus deren Existenz kann jedoch keine Inkonsequenz hinsichtlich der Vorstellungen über Kosmographie abgeleitet werden. Dieses Bild scheint im grossen und ganzen recht konsistent zu sein mit einigen wenigen Ausnahmen, die die Nordländer keineswegs weniger "logisch" in ihrer Kosmosauffassung machen als andere Völker. Dies wäre dagegen der Fall, wenn etwas so Wichtiges wie die Götter keinen festen Platz im Kosmos hätten.

Wie bereits vorher erwähnt, soll es hier keinesfalls darum gehen, die Analysen der herangezogenen Forscher zu schwächen, denn natürlich kann in der nordischen Mythologie von einer hoch-niedrig-Opposition mit äquivalenten Oppositionspaaren die Rede sein - das liegt im Begriff der vertikalen Achse an sich. Dagegen gibt es eine Reihe Details in diesen Analysen, die diskutiert werden sollten. Es dreht sich in erster Linie um die Konvertierung der Begriffe von dem einen "Kode" (Achse) zum anderen, wie wir es besonders bei Molenaar und Meletinskij gesehen haben; ein anderer Punkt ist die Beziehung $z$ wischen den beiden räumlich Modellen einerseits und den beiden zeitlichen Modellen andererseits, die bei Molenaar und Hastrup eine Rolle spielen.

"Strophe 18 des gleichen Gedichts, wo wir die Kenning "býskips bæe" finden können, ist kein endscheidendes Argument für eine himmlische Wolınstatt der Götter. Die Kenning ist unsicher, und ohne einen alternativen Vorschlag vorzubringen, scheint "die Wohnung der Luft" nicht überzeugend, worauf auch Finnur Jónsson aufmerksam macht (LP 73). 
Von einem Kode zum anderen "zu übersetzen" ist keineswegs unproblematisch. Molenaar zieht die Schlussfolgerung, dass Norden im horizontalen Modell und die Unterwelt im vertikalen Modell mit den gleichen semantischen Inhalten belegt sind. Als Beweis wird Snorri (Kap. 34) angeführt, wo Hermóðr, um nach Hel zu kommen, nach unten und nach Norden gehen muss. Snorri baut hier augenscheinlich auf älteren Vorstellungen von einem Todesreich im Norden und von den Riesen als Leichenesser auf ( $\mathrm{Vm}$ 37, HHj 16). Ob dies wirklich dazu berechtigt, die Behauptung aufzustellen, dass dieses Totenreich und Hel in heidnischer Zeit identisch waren, möchte ich noch bezweifeln. Andererseits kann man sich ohne weiteres vorstellen, dass der Weg nach $\mathrm{Hel}$ in nördliche Richtung führte, da es wesentlich natürlicher erscheint, den Norden mit seiner Kälte mit der Unterwelt unten in der Erde zu parallelisieren als mit einem glühendheissen Ort, wie man es von den christlichen Vorstellungen her kennt. Auf jeden Fall gibt es ausser Snorri keinen Beleg dafür, dass Hel in dem horizontalen Modell überhaupt einen Platz einnehmen kann ${ }^{7}$, wohingegen es zahlreiche Beispiele dafür gibt, dass man es sich als zur Unterwelt gehörig vorgestellt hat. Es kann natürlich nicht ausgeschlossen werden, dass eventuell eine Vorstellung über verschiedene Richtungen im horizontalen Sinne in der Unterwelt existiert haben kann, aber es wird in den Vorstellungen über sie kaum eine weitere Rolle gespielt haben. Hel war in erster Linie unten.

Die Frage stellt sich nun, ob wir - selbst wenn es eventuell ein Totenreich in nördlicher Richtung gegeben haben sollte - ohne weiteres behaupten können, dass die Unterwelt und Útgarðr in den beiden Kosmossystemen die gleiche Bedeutung haben. Dieser Meinung ist Molenaar offenbar und Meletinskij ist an dieser Stelle ganz explizit. Er sagt:

The vertical model [...] corresponds to the horizontal one by a series of identifications which are essentially transformations. The main hinge, tying and strengthening boths models is the identification of the "north" and also the east, with the below (the locus of the kingdom of the dead and generally of

7 Der Saal, dessen Tür nach Norden zeigt und den man auf Nástrond ( $V s p 38$ ) findet, kann nach Meinung Finnur Jónssons (LP 424) im nördlichen Teil von Hel liegen und demnach ein Teil davon sein. Dass es in Hel einen Strand gibt, hängt wohl natürlicherweise damit zusammen, dass der Weg dorthin oft über Wasser oder eine Brücke fülırt. Im übrigen wird an der Stelle nichts davon gesagt, dass der Saal in nördlicher Richtung liegt, nur dass die Tür nach Norden zeigt, was damit zusammenpasst, dass der Weg nach Hel von der Oberwelt zuerst nach Norden und danach nach unten geht. 
Chtonian demonic forces). The watery element in the horizontal model (the sea) figures essentially a negative quantity (sources, roots). Jormungandr is in some measure equivalent to Nidhögg who gnaws at the roots of the cosmic tree. In the vertical model there is no shaman mediation by Loki between the Aesir, the giants and the dwarfs and it is Odin who carries out the shaman functions. Only in the vertical model is the description of the heavenly world of the gods and of the heavenly "happy" Kingdom of the dead developed, but to make up for it, the opposition of gods and giants and the struggle with them is virtually absent. The opposition with the giants does to some extent correspond to the opposition with the Kingdom of the dead and the chtonian forces. Therefore, if the opposition "culture-nature" is most pronounced in the horizontal model, it is the opposition "cosmos" and "chaos" which is in the foreground in the vertical model (Meletinskij 1973a, 50).

Dies ist ausgesprochen interessant, aber die Frage stellt sich, ob es auch richtig ist. Zunächst gibt es, wie bereits oben angesprochen, Probleme mit der Analogie von "Norden" und "Osten" einerseits und der Unterwelt andererseits. "Norden" und "Osten" im horizontalen Modell sind vor allem als der Ort charakterisiert, wo sich die Riesen aufhalten und wo Pórr hinreist, um sie zu bekämpfen. In der Unterwelt gibt es, wie Meletinskij selbst sagt, praktisch keine Riesen ${ }^{8}$, dafür aber eine andere Kategorie von Wesen, deren dämonischer Charakter nicht

${ }^{8}$ Mímir könnte hier eine Ausnahme sein, denn was ihn betrifft, so ist er zweifellos mit der Unterwelt verbunden. Es ist schon eher diskutabel, ob er ein Riese ist. Dass sein Name in einem Pula als Riese vorkommt, scheint nicht überzeugend, zieht man die Rolle in Betracht, die er im Krieg der Wanen einnimmt, wie er in Yng.s Kap. 4 dargestellt wird und die Rolle, die er als Wissensvermittler für Odin spielt, ua., weil Odin sich durch seine Wissenswettsbewerbe als klüger als die Riesen erwiesen hat. Mímir kann ein Ase sein, der durch eine Reise in die Unterwelt oder das Totenreich sich ein Wissen angeeignet hat, was es nur dort gibt. Dass es sich auch um einen Riesen handeln kann, ist jedoch nicht ganz von der Hand zu weisen. ( $\mathrm{Vgl}$. Sokkmímir, u.a. in Yt. 2 und Grm 50 und andere Zusammensetzungen mit Mímir). Denn selbstverständlich können die Riesen, genau wie die Götter in die Unterwelt reisen. Das typische Beispiel ist Loki, aber seine eigentlich Wohnstatt liegt nicht dort. Etwas anders verhält es sich mit den Riesinnen, die - nicht primär als Riesen - sondern als Frauen, also als Ausdruck eines femininen Prinzips (das als der Unterwelt zugehörig klassifiziert wird, vgl. Hastrup 1981; Schjødt 1983), aber auch als Tote der Unterwelt angehören, denn Riesen sowohl als Menschen können nach Hel kommen, wenn sie sterben. Dass daher nicht nur die Volva in Bdr, sondern auch in Vsp und Helreið Brynhildar als Tote aufgefasst werden müssen, dafür habe ich bereits früher argumentiert (Schjødt 1981).

Dagegen kann ich Einar Haugens Vorschlag kaum akzeptieren, dass die Zwerge nur diminutive Riesen sein sollen (Haugen 1966, 863). Die Tatsache, dass sie gewisse Dinge mit ihnen gemeinsam haben, macht sie nicht $\mathrm{zu}$ solchen. 
unbedingt einleuchtend ist. Hel ist sicherlich keine besonders anziehende Persönlichkeit, aber das "Dämonische" an ihr, ist wiederum vor allem auf Snorris Beschreibung zurückzuführen (Gylf. Kap. 20), der nach allgemein anerkannter Auffassung christlich beeinflusst war, zumal alle sich einig sind, dass Hel kein Totenreich war, wo man Qualen ausstehen musste, sondern eher der klassischen Vorstellung von Hades entsprach - also einem Ort, der gewiss freudlos war, aber keineswegs einer eigentlichen Hölle gleichkam. Sieht man einmal von Hel selbst ab, so ist es dagegen schwierig, die Dämonie in der Unterwelt zu entdecken. Die Gruppen chthonischer Wesen, die oben erwähnt worden sind, haben gewiss ein grösseres Wissen über Magie und andere Künste als es in der Oberwelt der Fall ist, aber deshalb sind sie noch lange nicht mit den Riesen äquivalent, deren Relation zu Göttern und Menschen - trotz der ursprünglichen Verwandtschaft - eindeutig antagonistisch ist. Ich möchte also behaupten, dass die Opposition, von der auf der vertikalen Achse die Rede ist, eine ganz anderer Art ist, als diejenige, der wir auf der horizontalen Achse begegnen. Die Oppositionspaare auf den zwei Achsen weisen jedoch nicht nur einen unterschiedlichen Charakter auf, sondern sie finden auch bei ganz verschiedenen Kodes Anwendung. ${ }^{9}$

Mit Hinweis auf das Meletinskij-Zitat weiter oben kann man in den beiden Oppositionspaaren Kosmos vs Chaos und Kultur vs Natur den Ausgangspunkt nehmen. Diese kommen seiner Meinung nach am deutlichsten auf der vertikalen bzw. der horizontalen Achse zum Ausdruck. Es ist jedoch nicht besonders ersichtlich, warum sich das so verhalten sollte, und auf jeden Fall erfordert es ein Diskussion der Terme Chaos und Natur: was ist eigentlich damit gemeint, was sind deren semantische Konnotationen im Norden und wie verhalten sie sich zueinander? Diese Fragen müssen zwangslaufig beantwortet oder zumindest kommentiert werden, bevor man Meletinskijs Hypothese (oder Theorie?) zustimmen oder sie ablehnen kann. Dies ist jedoch aus Platzgründen nicht möglich, daher will ich mich mit einigen kurzen Bemerkungen begnügen, die den Gebrauch dieser Ausdrücke wenigstens problematisieren.

${ }^{9}$ Ich will natürlich nicht bestreiten, dass gewisse Transformationen zwischen den beiden räumlichen Modellen stattfinden können (z. B. Njørðr, der als ursprünglich clithonischer Gott in der Oberwelt später Bewohner der Gebiete am Meer wurde), aber es sind keine Transformationen, die Alternativen bewirken und folglich inkonsistent sind, wie es der Fall ist mit der Frage, ob die Götter im Himmel wohnen oder auf der Erde. Es ist im Gegenteil die Rede von Transformationen, die ganz in Übereinstimmung mit der narativen Eigenlogik der Mythen stehen. 
Nun verhält es sich so, dass Chaos im Verhältnis zu Kosmos und Natur im Verhältnis zu Kultur sowohl räumliche als auch zeitliche Implikationen hat. Gleichzeitig damit, dass man sie ausserhalb ihrer Gegenpole findet und dass man also zu ihnen "reisen" kann, so befinden sie sich auch "vor" ihren Gegenpolen: Kosmos entsteht aus dem Chaos und die Natur kann kulturähnlich gemacht werden. Ausserdem ist es wichtig, dass man sich klarmacht, dass beide Terme kulturbezogen sind: sie kömnen nur im Verhältnis zu dem semantischen Universum definiert werden, das die Kultur definiert. Das bedeutet wiederum, dass sie im wesentlichen nur als Oppositionen zu Kosmos bzw. Kultur definiert werden können. Nun verhält es sich so, dass jede Kultur sich als in Übereinstimmung mit der kosmischen Ordnung auffasst, weshalb Kosmos, definiert als das geordnete Universum, und Kultur sich zueinander verhalten wie Makrokosmos zu Mikrokosmos, da das letztere ein Abbild des ersteren ist. Auf einem gewissen Niveau ist es daher nicht möglich, zwischen Kosmos und Kultur zu unterscheiden: Die Strukturierungs- und Klassifikationsprinzipien, die für das eine gelten, gelten auch für das andere (vgl. die oben angeführte Argumentation bei Gurevich und Hastrup, über diese Prinzipien im Verhältnis zu Kosmologie und Gesellschaft). Wenn sich eine solche Unterscheidung mit den beiden markierten Termen Kultur und Kosmos nicht durchführen lässt, muss es folglich auch schwierig werden, die negativen Pole im Verhältnis zueinander zu definieren. Dieses Problem wird noch deutlicher, wenn man sich das nordische Material ansieht; in der nordischen Mythologie kommen kaum richtige Menschen vor, da die Protagonisten der meisten mythologischen Plots fast ausschiesslich Götter und andere übernatürliche Wesen sind. Dennoch würden die meisten Chaos wohl als ein zeitliches Phänomen definieren (etwas, das bereits dagewesen ist und evt. wiederkommen kann), während Natur dementsprechend als ein räumliches Phänomen verstanden wird (das, was die Kultur umgibt). Dieser Blickwinkel kann jedoch nicht konsequent durchgehalten werden, da es klar ist, wie wir oben gesehen haben, dass beide Terme (auf jeden Fall im Norden, vernutlich aber ïberall) zeitliche und räumliche Konnotationen aufweisen. Eine solche Unterscheidung ist daher nicht zweckmässig. Es scheint ergiebiger zu sein, sich die Zusammenhänge anzusehen, wo die Terme Chaos und Natur angemessenerweise in Übereinstimmung mit dem Bedeutungskomplex gebraucht werden können, den sie innerhalb der modernen anthroprologischen und religionshistorischen Literatur in sich tragen. Dann kann man sehen, dass wir es in allen Situationen, wo es natürlich wäre, den Term Chaos zu gebrauchen, mit Riesen 
zu tun haben. Es dreht sich in dem zeitlichen Kode vorrangig um Kosmogonie und Eschatologie. In der Kosmogonie, so wie wir sie von Snorri erzählt bekommen und wie sie bruchstückhafter in den übrigen Quellen vorkommt, spielen die Riesen eine alles dominierende Rolle: sie sind die Ältesten, und ihr Stammvater Ymir ist es, der das Baumaterial für den gesamten Kosmos liefert. In diesem Zusammenhang ist es wichtig, darauf zu achten, dass dieser Urriese erst nach seinem Tod Bedeutung erhält, als die Götter aus dem ursprünglichen Chaos den Kosmos erschaffen. Die Kosmogonie als solche kann daher als eine doppelte Opposition zwischen dem Chaos und den Riesen auf der einen Seite und dem Kosmos und den Göttern auf der anderen Seite analysiert werden. Das gleiche gilt für die Eschatologie, wo der endgültige Untergang auf den Kampf der Riesen mit den Göttern zurückführen ist, wobei die letzteren auf verschiedene Art und Weise immer als Beschützer und Schöpfer des geordneten Kosmos gegolten haben. Etwas Ähnliches gilt auch für den räumlichen Kode. Hier haben wir gesehen, dass genau das, was ausserhalb der bekannten Welt ist, was am äussersten plaziert, also am weitesten von den Behausungen der Götter und Menschen weg ist - das ist das Land der Riesen, das, nicht zuletzt durch den zeitlichen Kode, eine eindeutig negative Bedeutung erhält, was gerade charakteristisch für das Chaos ist. ${ }^{10}$ Es scheint daher völlig ohne Übereinstimnung mit den Quellen, zu behaupten, dass Chaos vs Kosmos auf der vertikalen Achse am deutlichsten hervortritt: Chaos droht ganz allein auf der horizontalen Achse.

Der Begriff Natur ist dagegen wesentlich schwieriger zu hantieren. Über Chaos kann man wenigsten sagen, das es negative Konnotationen in sich trägt, wohingegen dies bei der Natur nicht, oder auf jeden Fall nicht eindeutig der Fall ist. Die Natur ist natürlich etwas anderes als die Kultur, aber im Gegensatz zu der Opposition Kosmos-Chaos, ist hier nicht die Rede von Feindschaft, wenn die Götter (oder die

10 Deswegen erscheint es auch nicht überzeugend, wenn einige Forscher versucht haben, mythische Ausdrücke zu finden für den Versuch, ein Gleichgewicht $z$ wischen Kosmos und Chaos aufrechtzuhalten, wo auch die Kosmosmächte nicht die Überhand bekommen dürfen und so das Gleichgewicht zerstören. Dies gilt besonders im Verhältnis zu der Midgardsschlange (Boyer 1981, 194 ff.; Meulengracht Sørensen 1986). Denn die gesamte nordische Mythologie ist Ausdruck eines andauernden Kampfes, wo das Böse überwunden werden soll, und der Mensch oder "Mythenmacher" kann diesem Konflikt keineswegs neutral gegenüberstehen: es wäre natürlich ideal, wenn die Götter siegen würden, die Welt aber zeigt, dass dies nicht so gewesen ist. 
Menschen), das aufsuchen, was wir Natur nennen - verstanden als eine kulturell definierte semantische Kategorie. Aber was macht den semantischen Inhalt dieses Begriffs aus? Es muss leider konstatiert werden, dass hier eine gewisse Subjektivität mit hereinspielt, u.a., weil in der Religionsphänomenologie "Natur" ein Begriff ist, mit dem man sich nicht beschäftigt hat - vermutlich aus der Betrachtung heraus, dass "Natur" als objektive Grösse definierbar ist, was natürlich nicht zutrifft. "Chaos" ist dagegen eine Kategorie, die religionsphänomenologisch im Verhältnis zum Kosmos immer eine Rolle gespielt hat. Wir stehen daher in gewissem Sinne ganz am Anfang, wenn wir die Natur im Verhältnis zu dem semantischen System, in das die Kosmologie auch als Teil eingeht, definieren sollen. Es muss jedoch im Rahmen des Erlaubten liegen, die Natur als eine Welt zu betrachten, die ausserhalb der Kultur liegt, und die sich nicht an die Spielregeln hält, die für die Kultur gelten, jedoch ausgesprochen mächtige Kräfte zur Verfügung hat, deren sich gewisse Repräsentanten der Kultur besser zu bedienen wissen als andere, z. B. Frauen und die Toten, sowie natürlich Zauberer. Diese Kräfte werden also mehr oder weniger von gewissen Individuen der Gesellschaft beherrscht, und sie können sowohl negativ als auch positiv eingesetzt werden. Zeitlich gesehen ist die Natur genau wie das Chaos etwas, was "noch nicht" in Übereinstimmung mit den Regeln gebracht worden ist, die in der jeweiligen Kultur gelten; aber im Gegensatz zum Chaos wird diese Abweichung nicht vorrangig als etwas Negatives betrachtet. Es ist im Gegenteil von entscheidender Bedeutung, dass die Kräfte, die hier verborgen liegen, "kultiviert" werden. Daher sehen wir auch oft, während der Übergang von Chaos zu Kosmos sich in Form von Kampf ausdrückt, wo die Repräsentanten des Kosmos notwendigerweise siegen müssen, dass das Verhältnis zu den Repräsentanten der Natur eher auf friedlichem Austausch basiert. ${ }^{11}$

11 Dies bedeutet jedoch nicht, dass die Gegensätze im vertikalen Modell "schwächer" sind; sie sind nur von anderer Art. Man kann im Gegenteil sehen, dass die horizontalen Gegensätze quantitativer Art sind (der eine Teil ist "besser" als der andere), wohingegen die Oppositionen auf der vertikalen Achse wesentlich stärker qualitativ markiert sind: z. B. Leben vs Tod, maskulin vs feminin. Diese qualitativen Gegensätze gehören nicht desto weniger zusammen wie Yin und Yang, während die horizontalen Gegensätze (wo Götter und Riesen im übrigen miteinander verwandt sind) auf der Frage basieren, wer der Stärkste ist - aber mit einem gleitenden Übergang zwischen den Polen. Hier dreht es sich um böse oder gut, und der Unterschied ist, wie bekannt, nicht immer einsichtig, und das gilt auch für die alten Nordländer.

In diesem Zusammenhang ist es interessant, auf den Unterschied achtzugeben, den es zwischen den beiden wichtigsten Göttern in der Mythologie, Ódinn und 
Das hängt natürlich auch damit zusammen, dass Chaos als Negation zu Kosmos Ausdruck einer "Unordnung" sein muss, während die Natur nur Ausdruck einer "anderen Ordnung" ist. So gesehen stehen also Kultur und Natur in Opposition zu Chaos. Andererseits steht es jedoch ausser Zweifel, dass die Symbole, die Konnotationen wie "Unordnung" und "die andere Ordnung" hervorrufen, zusammenfallen können, da es sich in beiden Fällen um Negationen der "richtigen Ordnung", nämlich Kosmos und Kultur, handelt.

Wenn diese Hypothese stimmt, dann folgt daraus auch, dass gerade die Unterwelt im Norden und der Austausch, der zwischen ihr und der Oberwelt stattfindet, das "Naturelement" repräsentiert: hier geht der Austausch zwar nicht reibungslos vor sich, ist aber doch im allgemeinen eher durch komplementäre als konträre Gegensätze geprägt. Dies gilt im Bezug auf die Wanen, deren chthonischer Charakter wohl kaum bezweifelt werden kann, im Bezug auf die Zwerge, die auch Affinität zur Unterwelt haben, und nicht zuletzt im Bezug auf alles, was mit Wissensaneignung zu tun hat. Derartige Wissensaneignungen finden immer auf die Art und Weise statt, dass "die Repräsentanten der Oberwelt" entweder direkt oder indirekt mit der Unterwelt Kontakt aufnehmen oder mit den Toten (indem sie eventuell selber sterben). Dies ist sowohl in der Eddadichtung als anderswo der Fall, z. B. in Grógaldr, in Bdr, in der Mythe vom Erhängen in Háv, in den Mythen von Mímir, in den Mythen von Kvasir und anderen. "Natur" und die Unterwelt werden dadurch zum Ausdruck der "anderen Welt", wie wir es in der Religionsphänomenologie nennen - derjenigen Welt, die die potentiellen Kräfte beinhaltet, die in der Oberwelt, evtl. in Form von Magie, manifestiert werden können. Chaos ist seinerseits natürlich auch Ausdruck einer "anderen Welt", aber es ist eine Welt, deren Potential eindeutig destruktiv ist und folglich bis hin zu Ragnarok bekämpft werden muss.

$\mathrm{Ob}$ "Natur" in diesem Zusammenhang ïberhaupt ein guter Ausdruck ist, kann in hohem Masse diskutiert werden, aber das Obige hat auf jeden Fall die etwas oberflächliche Anschauung problematisiert,

Dórr, gibt, die offenbar jeweils mit ihrer Achse verbunden sind - nämlich der vertikalen bzw. der horizontalen. Óðinn's Reisen finden in den meisten Fällen längs der vertikalen Achse zur Unterwelt statt, während Dórr's an der horizontalen Achse entlang nach Útgarðr gehen. Während Pórr auf diese Weise den einen (den positiven) Pol in einem Oppositionspaar ausmacht, der auf gut vs böse basiert, wird Óðinn wesentlich mehr zusammengesetzt, weil es gerade seine primäre Funktion ist, das Potential, das in der Unterwelt liegt, an die Oberwelt zu bringen und zu aktualisieren. 
der Meletinskij Ausdruck verlieh, denn wenn die Opposition Natur vs Kultur überhaupt relevant ist, dann verhalten sich diese und die Opposition Chaos vs Kosmos ungekehrt im Verhältnis zu den beiden räumlichen Achsen.

Es ist auf gleiche Weise problematisch zu behaupten, dass die zwei zeitlichen Modelle (Reversibilität und Irreversibilität) jedes für sich an ihr räumliches Modell gebunden seien. "... the vertical model was explicitly concerned with the irreversability of time", sagt Hastrup (Hastrup 1981, 68), und spricht dann, als Beleg dafür, von Yggdrasill und dem Schicksal. Nun ist dieses Gebiet natürlich auch zu umfassend, um hier ausführlich diskutiert zu werden, aber die Aussage scheint nicht unmittelbar einleuchtend zu sein.

Zunächst einmal scheint die horizontale Achse nur in geringem Umfang überhaupt zeitliche Konnotationen zu haben. Es stimmt natürlich, dass die Kosmogonie, wie wir gesehen haben, vorzugsweise dort stattfindet, aber dariber hinaus kann man kaum behaupten, dass von einer Art Reversibilität die Rede sein könnte, nur weil unmittelbar keine Entwicklung stattfindet und die Kosmologie stabil bleibt. Im Gegenteil scheint gerade die Relation Götter vs Riesen für die Phasen im kosmischen Verlauf entscheidend zu sein, und diese Relation wird, wie gesagt, auf der horizontalen Achse konstituiert. Umgekehrt ist die vertikale Achse mit Yggdrasill als Hauptbestandteil nicht eindeutig irreversibel. Das Symbol des Baumes selbst ist, wie Eliade nachgewiesen hat (Eliade 1958, 265 ff.) oft ein Symbol der Wiederholung, der Rückkehr, der Fähigkeit wieder zu erblühen, und daher also der Reversibilität. Es stimmt, dass Yggdrasill nicht zuletzt in Vsp dazu gebraucht wird, die Zeit zu messen und die fortschreitende Zeit bis hin zu Ragnarok zu symbolisieren, aber wir stehen hier eher dem Verhältnis zwischen kleinen und grossen Verläufen gegenüber. Teilt man einen zyklischen Verlauf in kleinere Einheiten auf, so wird dieser linear, da er eine Entwicklung hin zu einem neuen Zustand darstellt. Ich habe früher bereits dafür argumentiert, dass wir Hinweise auf eine zyklische Zeitauffassung in Vsp selbst finden können (Schjødt 1981), und es scheint mir innerhalb einer Religion mit einer derart grundlegenden zyklischen Ideologie, nicht möglich, Reversibilität und Irreversibilität als Gegensatzpaar aufzustellen. Selbst in Indien, wo die zyklische Auffassung ausgesprochen deutlich und für das gesamte religiöse Universum konstituierend ist, sehen wir, dass die makrokosmische Zeit in Verläufe (Yugas) eingeteilt ist, wo jede ihren linearen Verlauf hat. Das Lineare ist also fast per Definition im Zyklischen eingebaut, und im Norden ist es vorzugsweise Yggdrasill, der mit seiner Entwicklung 
Exponent für diesen Teilverlauf ist, der unsere Zeit ausmacht. Das einzige konsequente Beispiel, das wir für Reversibilität innerhalb dieses Verlaufs im Norden haben, ist das Schicksal der toten Krieger in Walhall - mit deren Tod und Wiedergeburt; aber man kann nicht sagen, dass dieses Beispiel auf der horizontalen Achse stattfindet.

Wiederum wirkt es so, als ob die genannten Forscher einem Systemzwang zum Opfer gefallen sind, der nicht notwendig ist, um strukturalistische Analysen zu machen.

Zum Schluss will ich nur noch an ein wichtiges strukturalistisches Prinzip erinnern, das u.a. für Lévi-Strauss in seinem Buch über Totemismus entscheidend war, nämlich, dass die Identität zwischen verschiedenen Kodes sich auf Relationen und nicht auf Elemente bezieht. Dies führt für diese Untersuchung mit sich, dass himmlische Symbole ohne weiteres in Verbindung mit chthonischen oder anderen vorkommen können und dass es daher die Relation hoch vs niedrig sein kann, die hier expliziert wird. Aber es ist wichtig, daran festzuhalten, dass es Relationen und nicht Elemente sind, die bedeutungstragende Werte in sich tragen: ein himmlisches Symbol kann in Hinblick auf die vertikale Plazierung dazu gebraucht werden, es von einem chthonischen zu unterscheiden; das eine ist die Oberwelt, während das andere die Unterwelt ist. Wenn also der französische Forscher Renauld-Krantz z. B. auf diese Weise drei verschiedene Niveaus ausfindig macht, wo die drei Funktionen wiedergefunden werden können, und er in Verbindung mit der kosmischen Taxonomie bereits behauptet, dass die erste Funktion mit dem Himmel verbunden ist, so ist es keinesfalls beabsichtigt, dies hier zurückzuweisen. Man muss sich nur darüber im klaren sein, dass eine solche Feststellung nur dann einen Sinn ergibt, wenn zugleich die zweite und dritte Funktion mit der Luft bzw. der Erde verbunden ist: die drei Funktionen verhalten sich so zueinander wie die drei kosmischen Sphären sich zueinander verhalten (RenauldKrantz 1972, 215). Die wechselseitigen Relationen der Funktionen können somit in einer Reihe von verschiedenen Kodes wiedergefunden werden - wie z. B. dem kosmischen, aber auch dem sozialen und anthropologischen sowie auch auf verschieden anderen Ebenen. Dies hat jedoch nichts mit "Wohnstätten" weder im mythologischen noch im kosmologischen Sinne zu tun, sondern es bezieht sich ganz alleine auf einige Klassifikationsprinzipien.

Zum Schluss möchte ich noch gerne hervorheben, dass die hier im Artikel kritisierten Verfasser nicht deswegen kritisiert worden sind, weil ihre Werke unbrauchbar oder gleichgültig sind, sondern im Gegenteil, weil sie provozierend und dadurch ausgesprochen interessant 
sind - im Gegensatz zu manch anderem, das über dieses Gebiet verfasst worden ist. Ich kann mich daher dem übergeordneten Projekt, das diesen Untersuchungen zugrunde liegt, völlig anschliessen; aber eine fortlaufende Diskussion über diese Themen und eine Präzisierung der Terminologie und des Begriffapparates müssen notwendigerweise immer derartigen Versuchen folgen - und in diesem Zusammenhang soll mein Beitrag hier auch gesehen werden.

\section{Literat urverzeichnis}

Boyer, R. 1981. La religion des anciens Scandinaves. Paris.

Eliade, M. 1958. Patterns in comparative religion. New York.

Gurevich, A. Ya. 1969. Space and time in the "Weltmodell" of the old Scandinavian peoples. Mediaeval Scandinavia 2. Odense.

Hastrup, K. 1981. Cosmology and society in Medieval Iceland. Ethnologia Scandinavica. Lund.

Haugen, E. 1966. The mythical structure of the ancient Scandinavians: Some thougths on reading Dumézil. To honour of Roman Jacobson: Essays on the occasion of his Seventieth birthday, 11. October 1966 2. The Hague.

Holtsmark, A. 1964. Studier i Snorres mytologi. (Skrifter utg. av Det Norske Videnskaps-Akademi i Oslo 2, Hist.-Filos. Klasse, N. S. 4.) Oslo.

Kiil, V. 1960. Hliðskiálf og seiðhjallr. Arkiv för nordisk filologi 75. Lund.

Meletinskij, E. 1973a. Scandinavian mythology as a system 1. Journal of Symbolic Anthropology 1. The Hague.

- 1973b. Scandinavian mythology as a system 2. Journal of Symbolic Anthropology 2. The Hague.

Meulengracht Sørensen, P. 1986. Thor's fishing expedition. Words and objects. Ed. by G. Steinsland. Oslo.

Molenaar, H. 1985. Óđinns Gift. Leiden.

Motz, L. 1975. The king and the goddess. Arkiv för nordisk filologi 90 . Lund.

- 1980. Sister in the cave: The stature and the function of the female figures in the Eddas. Arkiv för nordisk filologi 95. Lund.

Neckel, G. 1913. Walhall. Dortmund.

Nordal, S. 1927. Völuspá. København.

Renauld-Krantz, P. 1972. Structures de la mythologie Nordique. Paris.

Schjødt, J. P. 1981. Völuspá - cyklisk tidsopfattelse i gammelnordisk religion. Danske Studier. Købenlavn.

- 1983. Livsdrik og vidensdrik. Religionsvidenskabeligt Tidsskrift 2. Århus.

- 1984. Aser og vaner: Historie eller struktur? Fallos 5. Århus.

Sijmons, B. \& Gering, H. 1906-31. Die Lieder der Edda 1-4. Halle.

Stanley Martin, J. 1972. Ragnarģk. Assen. 
Ström, $\AA$. V. 1975. Germanische Religion. Germanische und Baltische Religion. Von Å. V. Ström \& H. Biezais. (Die Religionen der Menschheit 19, 1.) Stuttgart.

Vries, J. de 1956-57. Altgermanische Religionsgeschichte 1-2. (Grundriss der germanischen Philologie 12, 1-2.) Berlin.

- 1964-67. Altnordische Literaturgeschichte 1-2. (Grundriss der germanischen Philologie 16.) Berlin. 